\title{
Entwicklung einer agil-strukturierten Prozesslösung mittels ASD
}

- Agile Systems Design für das technische Änderungsmanagement im After Sales eines OEM der Automobilindustrie

Jonas Heimicke, Sascha Pfau, Linda Vetten, Albert Albers

Die Anwendung agiler Arbeitsweisen rückt zunehmend in den Interessensfokus unterschiedlicher Bereiche in produzierenden Unternehmen. Eine erhöhte Reaktionsfähigkeit auf unvorhergesehene Veränderungen im Kontext, Steigerung der Transparenz bezüglich Arbeitsinhalt und Auslastung im Entwicklerteam sowie das erschaffen und Vorantreiben einer übergeordneten Teamvision und damit verbundene Performancesteigerungen sind nur einige der positiven Effekte, die hierdurch erzielt werden sollen. Da die genutzten Ansätze jedoch meist auf Best Practices aus der Branche der Softwareentwicklung resultieren, stellen sie die Bereiche in produzierenden Unternehmen vor neue Herausforderungen, wie den Umgang mit Normen und Standards im agilen Arbeiten, Datenschutz bei der Erfassung von Arbeitsaufwänden und den Umgang mit physischen Produkteigenschaften. Damit die Einführung von Agilität in die Abläufe produzierender Unternehmen nachhaltig erfolgt, wurde eine Methodik entwickelt, mittels derer ein individueller Prozess bestehend aus agilen und strukturierenden Elementen abgeleitet werden kann. Im vorliegenden Beitrag soll diese Methodik hinsichtlich ihrer Zweckmäßigkeit untersucht werden, um daraus in zukünftigen Forschungsarbeiten eine Weiterentwicklung dieser Methodik abzuleiten. Dies erfolgt in einem realen Anwendungsfall - dem Änderungsmanagement eines deutschen OEMs in der Automobilindustrie. Hierzu wurde der hier existierende Prozess beobachtet und erfasst, Interviews mit betroffenen Mitarbeitern geführt und Potentiale für eine Prozessverbesserung durch die Integration agiler Elemente definiert. Diese wurden im Anschluss ebenfalls im Zuge der Methodik zur Entwicklung der individuellen agilen Prozesslösung durch eine zum Anwendungsfall passende Gesamtmethode realisiert. Die Evaluation des Vorgehens hat gezeigt, dass der Anspruch der Individualität der entwickelten Gesamtmethode gegeben ist, jedoch noch kleinere Verbesserungspotentiale bestehen.

Keywords: Agile Prozesse, ASD - Agile Systems Design, Advanced Systems Engineering, integrierte Produktentwicklung, Änderungsmanagement, Case-Study. 


\section{Einleitung}

Das Umfeld von Unternehmen ist durch marktseitige und technologische Veränderungen geprägt. Diese Instabilität führt gerade bei produzierenden Unternehmen mit der zunehmenden digitalen Vernetzung von einzelnen Systembestandteilen zu einem Anstieg der Komplexität. In der Produktentwicklung resultieren die individuellen Anforderungen der Kunden sowie die Dynamik im Unternehmensumfeld in Unsicherheit und eine Vielzahl an Produktfunktionen (Frenz, 2020, S. 472). Dies führt unweigerlich zu einer Belastung bestehender betrieblicher Prozesse und Strukturen (Jania, 2004). Somit wird bei Prozessverbesserungen vermehrt Flexibilität und eine erhöhte Reaktionsfähigkeit gefordert. Für einen OEM der Automobilindustrie werden diese Anforderungen in einem Prozess des technischen Änderungsmanagements betrachtet. Um auch in Zukunft bei immer komplexer werdenden technischen Änderungen im After Sales wettbewerbsfähig zu bleiben, bedarf es einer Anpassung des Prozesses und einer Handlungsempfehlung zur Steigerung der Reaktionsfähigkeit im Umgang mit technischen Änderungen. Hierbei wird der ASD - Agile Systems Design Ansatz zur Entwicklung einer Gesamtmethode verwendet, wodurch sowohl strukturierende als auch flexible Elemente bedarfsgerecht implementiert werden sollen.

\section{Stand der Forschung}

\section{After Sales und technisches Änderungsmanagement}

Der Bereich After Sales umfasst die Tätigkeiten eines Unternehmens für Produkte und Dienstleistungen innerhalb der Nutzungsphase (Dimitrova, 2009, S. 114). Hierzu gehören alle Maßnahmen zur Beziehungspflege und der Servicepolitik nach dem Verkauf des Produktes (Schütze, 1994, S. 5). Demzufolge reicht die Zuständigkeit von den Serviceleistungen über die Kundenbetreuung bis hin zum Verkauf von Zubehör (Dimitrova, 2009, S. 114). Zusätzlich spielt die Ersatzteilversorgung im After Sales eine wichtige Rolle (Baader, Montanus \& Sfat, 2006). Das technische Änderungsmanagement steht allgemein für ein interdisziplinäres Management von technischen Änderungen (Langer, 2016, S. 524-526).

\section{Plangetriebene und agile Ansätze in Prozessen der Produktentwicklung}

Die plangetriebenen Prozesse sind allgemein durch einen sequenziellen Ablauf mit getrennten Phasen und einem definierten Ergebnis am Ende jeder Phase charakterisiert. Hiermit können primär die geforderten Ergebnisse geplant und der Prozess für den Kunden verständlich gemacht werden. Des Weiteren zeichnen sich plangetriebene Prozess dadurch aus, dass diese beliebig wiederholt und verbessert werden können. 
(Siebler-Guth, 2019, S. 19-20) Hierfür bekannt sind das Wasserfallmodell (vgl. Jakoby, 2015, S. 126-127) sowie der Stage-Gate Ansatz (Cooper, 1990).

Im Gegensatz zu plangetriebenen Ansätzen gibt es eine Vielzahl an agilen Ansätzen (Böhm, 2019, S. 29). Die gängigsten agilen Methoden in der industriellen Anwendung sind Scrum und Kanban. Eine transparente Darstellung und Analyse der Ist-Situation sowie hieraus abgeleitete Verbesserungen sind Teil des empirischen Vorgehens beider Methoden. Scrum und Kanban unterscheiden sich jedoch wesentlich in ihrer Vorgehensweise. (Kusay-Merkle, 2018, S. 34) Während Scrum eine festgelegte Zeitdauer für Sprints vorgibt, begrenzt Kanban den Arbeitsfluss durch eine Limitierung (KusayMerkle, 2018, S. 44-45).

\section{Methode zur Entwicklung und Einführung agiler Ansätze}

Nach Albers können Organisationen mittels einer erhöhten Agilität im Prozess auf Unsicherheiten in dynamischen Märkten reagieren (Albers, Heimicke et al., 2018). Hierbei muss jedoch für die jeweilige Situation ein praktikables Gleichgewicht zwischen flexiblen und strukturierenden Elementen erreicht und das Wissen der Organisation mit einbezogen werden (Reiß, 2018, S. 44). Das Gesamtkonzept ASD - Agile Systems Design verfolgt somit das Ziel „[...] situations- und bedarfsgerechte Methoden und Prozesse für die reale, praktische Anwendung zu erforschen, weiterzuentwickeln und zu implementieren" (Albers et al., 2019).

Die neun Grundprinzipien des ASD - Agile Systems Design sind Leitwerte, an denen sich im Unternehmen oder im spezifischen Anwendungsfalls bei der Entwicklung einer methodischen Unterstützung orientiert werden kann: (Albers et al., 2019; Albers, Heimicke, Trost 2020)

1. Der Mensch steht im Zentrum der Produktentstehung

2. Jeder Produktentstehungsprozess ist einzigartig und individuell

3. Agile, situations- und bedarfsgerechte Kombination strukturierender und flexibler Elemente

4. Jedes Prozesselement lässt sich im ZHO-Modell verorten und jede Aktivität basiert auf den Grundoperatoren Analyse und Synthese

5. Alle Aktivitäten der Produktentwicklung sind als Problemlösungsprozess zu verstehen

6. Jedes Produkt wird auf Basis von Referenzen entwickelt

7. Produktprofile, Invention und Markteinführung bilden die notwendigen Bestandteile des Innovationsprozesses 
8. Frühe und kontinuierliche Validierung dient dem kontinuierlichen Abgleich zwischen Problem und dessen Lösung

9. Für eine situations- und bedarfsgerechte Unterstützung in jedem Entwicklungsvorhaben müssen Denkweisen, Methoden und Prozesse skalierbar sein

Eine methodische Unterstützung soll bei der Entwicklung einer bedarfsgerechten Prozesslösung zur Integration von Agilität verwendet werden. Diese Methode folgt der SPALTEN-Problemlösungsmethodik (Albers, Reiß, et al. 2016), wobei die Erkenntnisse der Situationsanalyse (S) und der Problemeingrenzung $(P)$ in ein Methodenprofil resultieren. In der Suche alternativer Lösungen (A) werden dann für die in S\&P identifizierten Handlungsfelder maßgeschneiderte agile und strukturierende methodische Elemente vorgeschlagen. (Albers, Heimicke, Trost \& Spadinger, 2020)

\section{Grundlagen der Methode}

Nach Hales \& Gooch (2004) können für den ingenieurwissenschaftlichen Designkontext fünf Gruppen unterschieden werden: Makroökonomie, Mikroökonomie, Unternehmen, Management, Projekt und Individuum (Albers et al., 2020). Zur Beschreibung und Kategorisierung von Einflussfaktoren auf die agilen Fähigkeiten von Organisationseinheiten wird zunächst in zwei Level unterschieden. Hierbei werden nach Gericke et al. die beschriebenen Gruppen nach Hales \& Gooch (2004) als Level 1 - Ebene bezeichnet und in der Level 2 - Ebene durch insgesamt 35 Untergruppen weiter konkretisiert (Abbildung 1) (Gericke, Meißner \& Paetzold, 2013). Nach Albers werden die Gruppen Makroökonomie und Mikroökonomie der Level 1 - Ebene nicht weiter betrachtet. Denn diese beiden Gruppen können bei der Entwicklung von Methoden, die eine Kombination strukturierender und flexibler Elemente realisiert und ein anwendungsfallgerechtes Maß an Agilität in den Prozess bringt, nicht direkt beeinflusst werden. Die Gruppe Management auf der Level 1 - Ebene enthält z.B. auf der Level 2 - Ebene die Untergruppen: Werte, Führungsstil, Führungskompetenz, Unternehmensleitung (Albers et al., 2020). Auf dieser Struktur aufbauend wurden relevante Einflussfaktoren auf die agilen Fähigkeiten der Organisationseinheiten nach Albers für die 32 Untergruppen identifiziert. Über 200 identifizierte Einflussfaktoren können somit sowohl einer Level 1 - Gruppe als auch einer Level 2 - Untergruppe zugeordnet werden. Diese erarbeitete Sammlung von Faktoren dient bei der Entwicklung einer spezifischen Prozesslösung als Referenz für die Identifikation relevanter Einflussfaktoren. (Albers et al., 2020). 


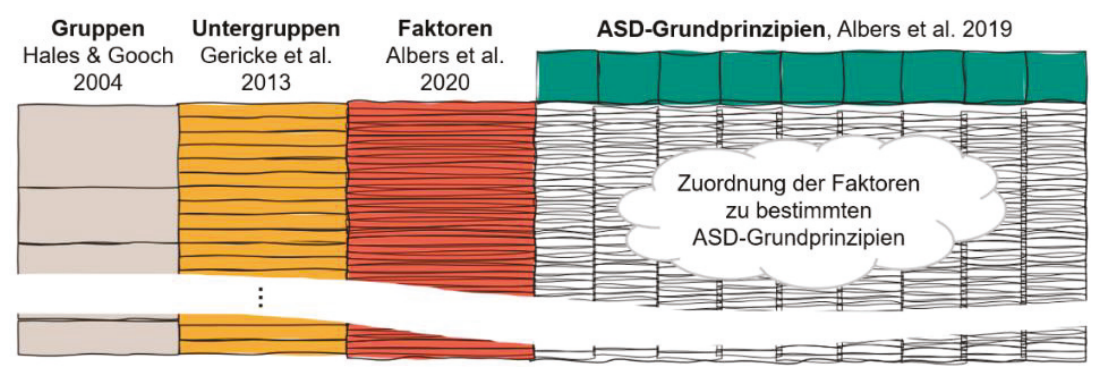

Abbildung 1: Verbindung der Einflussfaktoren mit den ASD-Grundprinzipien (in Anlehnung an Albers et al., 2020)

In einem weiteren Schritt wurden alle Einflussfaktoren den ASD-Grundprinzipien zugeordnet, auf welche durch den Einflussfaktor ein positiver Effekt erwartet wird. Hierbei ist jeder Faktor mindestens einem Grundprinzip zugeordnet, sodass die Grundprinzipien auch unterschiedlich viele Faktoren beeinflussen können. Jedoch müssen nicht alle Faktoren zwangsweise für jede Situation relevant sein. (Albers et al., 2020)

\section{Systematische Auswahl der ASD - Grundprinzipien und der Einflussfaktoren}

Um im Anwendungsfall den jeweils geeigneten Grad an Agilität umzusetzen und den angestrebten Effekt der Einflussfaktoren nutzbar zu machen, muss eine systematische Auswahl der relevanten Einflussfaktoren für das Zielsystem getroffen werden. Der Anwender durchläuft in der Methodendurchführung die folgenden Schritte: Identifizierung der eigenen Ist-Situation, Identifizierung geeigneter Handlungsfelder, Priorisierung der Grundprinzipien, Vorschlag geeigneter Faktoren. (Albers et al., 2020) Alternativ kann die Gewichtung der Handlungsfelder auch zunächst als Filter zur gezielten Auswahl von Einflussfaktoren verwendet werden. Hierdurch kann die situationsspezifische Relevanz der ASD-Grundprinzipien auf Basis der Gewichtung der gewählten Einflussfaktoren bestimmt werden. (Heimicke, Dühr et al., 2021; Heimicke, Ng, Krüger \& Albers, 2021) Somit kann mit Hilfe dieser Einflussfaktoren der gezielte Einsatz agiler Elemente unterstützt, und in einem nächsten Schritt methodische Elemente zur Realisierung der ASD- Grundprinzipien ausgewählt werden, sodass die Gewichtung erreicht wird. (Albers et al., 2020; Heimicke, Dühr, Krüger, Ng \& Albers, 2021)

\section{Forschungsdesign}

Die traditionellen Organisationsstrukturen in Unternehmen geraten durch sich häufig ändernde Marktanforderungen zunehmend an ihre Grenzen. Agilität erregt durch die 
in der Softwareentwicklung entstandenen Methoden auch in Unternehmensprozessen produzierender Unternehmen und im Projektmanagement vermehrt Interesse. Diese Ansätze können aus der Softwareentwicklung jedoch meist nicht auf andere Anwendungsbereiche übertragen werden und müssen auf den jeweiligen Kontext angepasst werden, denn längst sind nicht alle Methoden für jeden Anwendungsfall sinnvoll. Primär werden eine Verbesserung der Reaktionsfähigkeit und mehr Flexibilität in Prozessen gefordert. Eine methodische Unterstützung zur situations- und bedarfsgerechten Implementierung agiler Fähigkeiten bietet hierfür der ASD - Agile Systems Design Ansatz.

Es soll die Anwendbarkeit des methodischen Vorgehens zur Erarbeitung einer individuellen Prozesslösung durch einen spezifischen Anwendungsfall evaluiert werden. Für diese Arbeit ergeben sich hieraus die folgenden zwei Forschungsfragen:

Forschungsfrage 1: Wie gestaltet sich das Methodenprofil einer agilen Methodik für das technische Änderungsmanagement?

Forschungsfrage 2: Welche Implikationen für das methodische Erarbeiten individueller Prozesslösungen lassen sich aus dem vorliegenden Anwendungsfall ableiten?

Zur Evaluierung des methodischen Vorgehens wird eine empirische Forschung mit qualitativen Forschungsmethoden durchgeführt. Das technische Änderungsmanagement im After Sales eines OEM in der Automobilindustrie wird für den Anwendungsfall als Untersuchungsumgebung definiert. Durch Beobachtungen und Interviews mit Experten wird ein umfangreiches Problemverständnis für den Prozess der Untersuchungsumgebung aufgebaut. Alle relevanten Informationen werden anschließend in einem Methodenprofil zusammengefasst und bilden den Bedarf für die Entwicklung einer Gesamtmethode für die Untersuchungsumgebung ab. Mit Hilfe der erarbeiteten Anforderungen werden gezielt strukturierende und flexible Methoden und methodische Elemente identifiziert. Durch eine sinnvolle Kombination der Elemente im Einklang mit dem Methodenprofil wird ein spezifisches Prozessmodell für die Untersuchungsumgebung abgeleitet. Abschließend wird das methodische Vorgehen evaluiert. Hierfür wird die Methode zur Erarbeitung der individuellen Prozesslösung in einem Experteninterview reflektiert. Aus den gesammelten Erkenntnissen resultiert eine Beschreibung von Anforderungen an die Methode. Diese Auswertung zeigt sowohl den erreichten Umfang der Methode für den Anwendungsfall als auch die möglichen Verbesserungspotenziale auf. 


\section{Ergebnisse}

Im Rahmen einer Situationsanalyse der Untersuchungsumgebung wird sowohl der hierarchische Aufbau als auch der Ist-Prozesses erfasst. Darüber hinaus werden die betroffenen Stakeholder identifiziert und analysiert. Ergänzt wird die Betrachtung durch das sächliche Umfeld. Die Beziehung zwischen den Ursachen und Wirkungen helfen anschließend die Informationen der Situationsanalyse zu verdichten und eine erste Zielsetzung für die erkannten Probleme zu erarbeiten. Durch das aufgebaute Situationsverständnis können in der hierauf folgenden Problemeingrenzung die Funktionen der Einflussfaktoren und ASD-Grundprinzipien nach Albers et al. abgeleitet werden. Die für den Prozess relevanten Gruppen nach Hales \& Gooch (2004) und Untergruppen nach Gericke et al. (2013) werden durch einen Anwender der Untersuchungsumgebung in einem paarweisen Vergleich gewichtet und dienen als Filter für die Bestimmung der Einflussfaktoren nach Albers, sowie für eine erste situationsspezifische Relevanz der ASD-Grundprinzipien (Abbildung 2).

Die Beschreibung der Einflussfaktoren sowie das angeeignete Situations- und Prozessverständnis helfen dabei eine Vorauswahl von 46 Einflussfaktoren zu treffen. Hieraus soll der Anwender die relevanten Faktoren auswählen. Der Umfang soll hierbei 20 Faktoren nicht übersteigen.
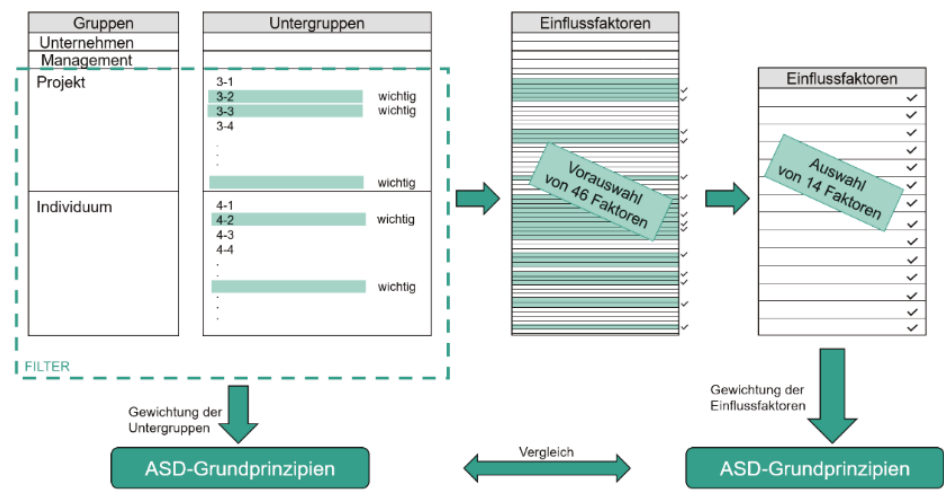

Abbildung 2: Ableitung der Einflussfaktoren

Analog der Gewichtung der Untergruppen im Filter wurden 14 gewählte Einflussfaktoren von dem Anwender in einem paarweisen Vergleich gewichtet. Hierfür wurde dem Anwender die Frage gestellt, bei welchem der jeweiligen verglichenen Einflussfaktoren das größere Verbesserungspotenzial für agile Fähigkeiten im Prozess gesehen wird. 
Anschließend wurden konkrete Funktionen und Idealzustände für die gewählten Einflussfaktoren bestimmt und zur Validierung mit dem Anwender auf weitere Faktoren und die Einhaltung der Randbedingungen geprüft. Mit Hilfe der Gewichtung der Einflussfaktoren und den jeweiligen zugeordneten ASD-Grundprinzipien wurde erneut die situationsspezifische Relevanz der ASD-Grundprinzipien berechnet und nachgeschärft.

Hierdurch kann die erste Annahme basierend auf der Gewichtung der Untergruppen des Filters durch einen Abgleich mit der Gewichtung der Einflussfaktoren überprüft werden. Die situationsspezifische Relevanz der ASD-Grundprinzipien ändert sich hierbei, wie in der Abbildung 3 ersichtlich, durch die Auswahl und Anpassung der Einflussfaktoren.

Durch das erarbeitete Problemverständnis können die Ziele abgeleitet und alle Ergebnisse aus der Situationsanalyse und der Problemeingrenzung in einem Methodenprofil als Anforderung an eine Gesamtmethode für die Untersuchungsumgebung formuliert werden.

ASD-Grundprinzipien des Filters

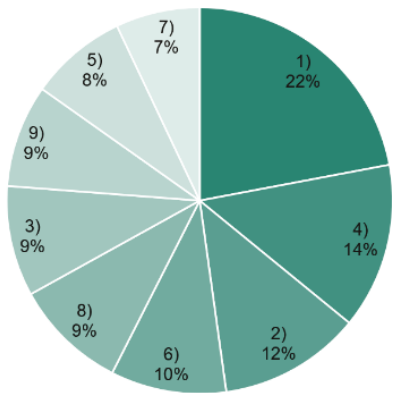

-1) $=4)=2)=6)=8)=3(-9)=5)=7$ )
ASD-Grundprinzipien der Einflussfaktoren

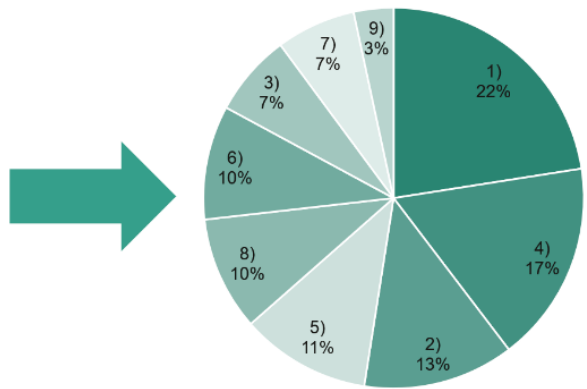

$-1)=4)=2)=5(-8)=6)=3(-7)=9$ )

1) Der Mensch steht im Zentrum der Produktentstehung

2) Jeder Produktentstehungsprozess ist einzigartig und individuell

3) Agile, situations- und bedarfsgerechte Kombination strukturierender und flexibler Elemente

4) Jedes Prozesselement lässt sich im ZHO-Modell verorten und jede Aktivität basiert auf den Grundoperatoren Analyse und Synthese

5) Alle Aktivităten der Produktentwicklung sind als Problemlösungsprozess zu verstehen

6) Jedes Produkt wird auf Basis von Referenzen entwickelt

7) Produktprofile, Invention und Markteinführung bilden die notwendigen Bestandteile des Innovationsprozesses

8) Frühe und kontinuierliche Validierung dient dem kontinuierlichen Abgleich zwischen Problem und dessen Lösung

9) Für eine situations- und bedarfsgerechte Unterstützung in jedem Entwicklungsvorhaben müssen Denkweisen, Methoden und Prozesse skalierbar sein

Abbildung 3: Situationsspezifische Relevanz der ASD-Grundprinzipien 


\section{Methodenprofil}

Die Gesamtmethode für die Untersuchungsumgebung ist als eine Prozesslösung zu verstehen, welche die Arbeitsabläufe in Richtung der im Verlauf der Methodenentwicklung identifizierten Ziele optimiert. Das Methodenprofil fasst die Ergebnisse zusammen und bildet zunächst die Basis für alternative Lösungen (A) sowie im weiteren Verlauf für eine Lösungsauswahl (L) der Prozesslösung.

Der Bedarf wird in einem Methodenclaim zusammengefasst und lässt sich für den Anwendungsfall folgendermaßen formulieren: „Wir brauchen eine Prozess/ösung, die den technischen After Sales im situationsgerechten Umgang mit technischen Änderungen unterstützt. Hierzu sollen agil-strukturierende Elemente die Aktualität von Ersatzteilen verbessern". Weiter lassen sich für die Erarbeitung einer Gesamtmethode folgende initiale Kernziele definieren:

— Individuelle Prüfung von Änderungsanträgen vermeiden

- Selbstorganisation fördern

- Transparenz im Prozess steigern

- Änderungsanträge priorisieren

- Dokumentation verbessern

— Skalierbarkeit zur Übertragung auf weitere Baureihen muss gegeben sein

Die Anforderungen an die Methode zur Entwicklung einer Gesamtmethode für die Untersuchungsumgebung wird durch bestimmte Rahmenbedingungen eingeschränkt. Zum einen müssen die Standards und Normen der Untersuchungsumgebung eingehalten werden. Weiter stehen nur die aktuellen Mitarbeiter mit dem gegebenen Kompetenzprofil zur Verfügung. Ebenfalls eingegrenzt wird die Methodenentwicklung durch etablierte Nachbarprozesse und eine gegebene Systemlandschaft. Die internen Ansprechpartner sind ebenso, wie die bestehende Organisationsstruktur, klar definiert. Weiter wird der Nutzen einer methodischen Prozessverbesserung für die drei Bereiche Anbieter-, Anwender- und Kundennutzen differenziert und formuliert.

\section{Ausgestaltung der Methode}

Im Rahmen der Methodenentwicklung wurde eine individuelle sowie situations- und bedarfsgerechte Prozesslösung erarbeitet. Hierfür wurden agile und strukturierende Elemente benötigt, welche die Ablauforganisation durch methodische Komponenten unterstützen können. Mithilfe der fallspezifischen Relevanz der ASD-Grundprinzipien und der Einflussfaktoren, mit deren Funktionsbeschreibung als kontextspezifische 
Wirkung, wurde eine Methodensammlung zur Entwicklung einer Gesamtmethode für die Untersuchungsumgebung zusammengestellt. Die Struktur sowie der In- und Output des Ist-Prozesses soll als Prozesswissen bestmöglich genutzt werden. Unter Berücksichtigung der Rahmenbedingungen sollten sowohl strukturierende als auch agile Elemente in den Prozess implementiert werden, sodass spezifische Aktivitäten und deren Zuständigkeiten benannt sowie bedarfsgerechte Methoden empfohlen werden können. Hierfür wurden Templates und Excel-Makros als rein strukturierende Elemente verwendet. Diese wurden mit agilen Elementen - wie bspw. dem Kanban-Board, dem Burndown-Chart und der Starfish-Methode - kombiniert, sodass der angestrebte Grad an Flexibilität für die Untersuchungsumgebung erzielt werden konnte. Um den Soll-Prozess transparent darzustellen, wurde aus dem iPeM integrierten Produktentstehungsmodell ein Prozessmodell für den spezifischen Anwendungsfall abgeleitet, welches dem Prozessverständnis des iPeM folgt (siehe Abbildung 4).

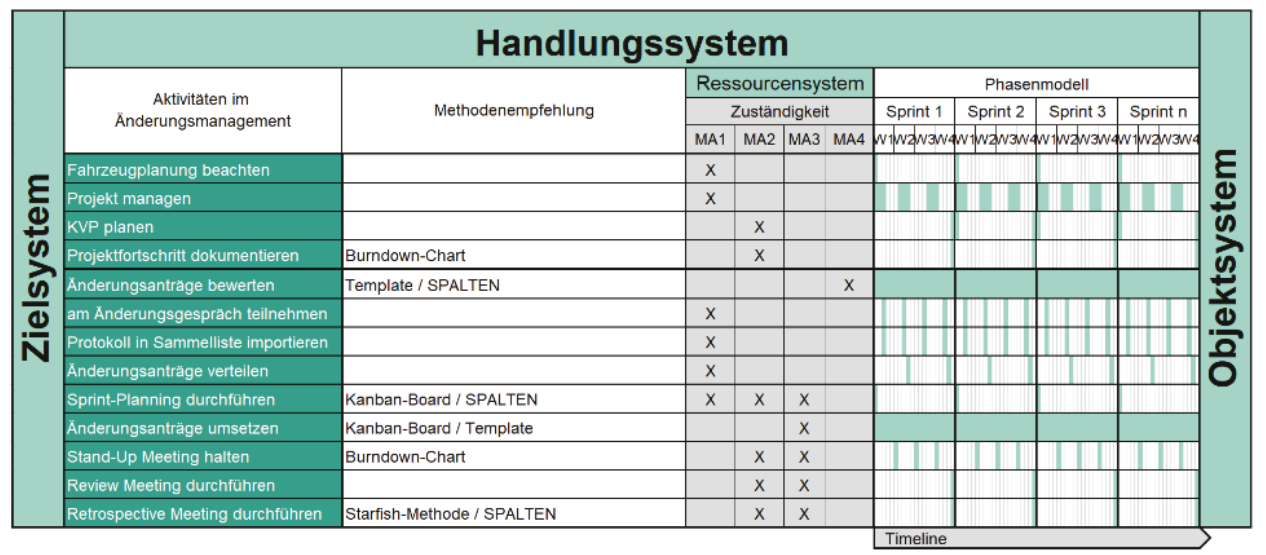

Abbildung 4: Spezifisches Prozessmodell (vgl. in Anlehnung an Albers et al., 2016)

\section{Evaluation}

Die Entwicklung einer methodischen Unterstützung ist ein dynamischer Prozess und beruht auf sich verändernden Annahmen. Die Auswirkungen und teilweise unbekannten Effekte können meist erst während des Entwicklungsprozesses festgestellt werden. Hierfür soll eine frühe und kontinuierliche Methodenevaluierung über alle Entwicklungsstufen erfolgen. Nach Blessing \& Chakrabarti (2009, S. 182-185) kann zur Evaluierung in Application-, Support- und Success Anforderungen unterschieden werden. Für die Identifikation und Einordnung der Anforderungen in die Bereiche Application, 
Support und Success wurden in einem Experteninterview gezielte Fragen gestellt. Die folgenden Ergebnisse fassen die wichtigsten Erkenntnisse und Antworten des Anwenders aus dem Experteninterview zusammen.

Frage: Denken Sie, ohne die Vorgehensweise wären vergleichbare Ergebnisse möglich gewesen?

Antwort: "Nein, glaube ich definitiv nicht, deswegen, weil es sich [...] um ein komplexes Problem handelt. [...] Aber in dem Ausmaß, wie es jetzt aufgezeigt worden ist und auch mit der Nachhaltigkeit, die das Ganze mit sich bringt, sicherlich nicht vergleichbar. Und dadurch, dass wir jetzt mit dieser Methode und ja, mit dem ganzen Aufbau den Output sozusagen begründet da liegen haben, haben wir natürlich eine viel bessere Argumentationsfähigkeit für Veränderungen im Team."

\section{Frage: Hat Ihnen im Vorgehen etwas gefehlt?}

Antwort: „[...] im klassischen Sinne in der Methodik selber, würde mir helfen, wenn man da irgendwie so eine klare Nennung von erreichten Teilzielen hätte, damit man den Fortschritt und auch den Erfolg des Projekts schon vor dem finalen Abschluss beobachten kann bzw. da auch irgendwie eine Berichtsform gegenüber der Führungsebene hätte."

Frage: Sehen Sie durch das individuell entwickelte Vorgehen,

das in diesem Projekt erarbeitet wurde, einen Mehrwert

gegenüber herkömmlichen agilen Methoden (z.B. Scrum)? Warum bzw. warum nicht?

Antwort: "Und das [...] angewendete Vorgehen ist individuell auf uns bzw. auf notwendige Verbesserung zugeschnitten und hat uns auch im Prozess die Möglichkeit gegeben, wirklich Pflöcke reinzuschlagen, wo wir gesagt haben, das ist unveränderlich, und die Methodik ist hier so flexibel, dass somit sichergestellt werden kann, dass die Theorie und die Praxis trotzdem noch im Einklang sind. Zusätzlich vermute ich ganz stark, dass wir durch die Methodik hier eine viel schnellere Umsetzbarkeit und Anwendbarkeit erreichen [...]."

Um die kritischen Punkte und den aktuellen Stand für weitere Verbesserungen und Maßnahmen aus dem Experteninterview ersichtlich zu machen, sind die Anforderungen farblich markiert (Abbildung 5). Alle Anforderungen, die durch die Methode im Anwendungsfall umgesetzt werden, sind grün hinterlegt. Die beiden Anforderungen (orange), dass die Methode „klar und verständlich“ aufgebaut sein soll und die "Betreuung durch Experten notwendig" ist, sind teilweise umgesetzt und können durch die händische Durchführung der Methode begründet werden. Eine toolseitige Unterstützung kann hierbei hilfreich sein und zur Umsetzung der Anforderung beitragen, jedoch ist ein Experte für die Erarbeitung einer individuellen Prozesslösung sinnvoll. 
Zwei weitere Anforderungen der Tabelle (rot) werden für den Anwender durch die Methode aktuell nicht umgesetzt. Zum einen wird gefordert, dass der Projektfortschritt bei der Anwendung der Methode erkennbar und ein Reporting möglich ist. Zum anderen müssen relevante Kriterien messbar gemacht werden. Die Qualitätskriterien sind aber in der Regel anwendungsfallspezifisch und müssen individuell erarbeitet werden. Alle nicht markierten Anforderungen (weiß) können zu diesem Zeitpunkt nicht bewertet werden.

\begin{tabular}{|l|l|l|}
\hline Application & Support & Success \\
\hline Klar und verständlich & Spezifisches Wissen generieren & Relevante Kriterien messen \\
\hline $\begin{array}{l}\text { Sinn der Arbeitsschritte } \\
\text { kommunizieren }\end{array}$ & $\begin{array}{l}\text { Argumentation ist belegbar } \\
\text { - Anerkennung und Akzeptanz }\end{array}$ & Definition von Arbeitspaketen \\
\hline $\begin{array}{l}\text { Betreuung durch Experten } \\
\text { notwendig }\end{array}$ & $\begin{array}{l}\text { Projektfortschritt und Reporting } \\
\text { muss möglich sein }\end{array}$ & Flexibilität ermöglichen \\
\hline Zielorientierung & Nachhaltigkeit erreichen & Kapazität nutzbar machen \\
\hline Skalierbarkeit & Komplexität verringern & Randbedingungen einhalten \\
\hline
\end{tabular}

Abbildung 5: Anforderungen an die Methode

Abschließend stellt der Anwender fest, dass das Vorgehen aus dessen Sicht immer dann sinnvoll ist, „wenn es darum geht etablierte Prozesse begründet zu hinterfragen und einer nachhaltigen Optimierung auf die Schliche zu kommen“. Dies spricht für einen hohen Stellenwert der methodischen Herangehensweise für eine Prozesslösung, die eine intensive und strukturierte Betrachtung erforderlich macht. Der Anwendungsfall hat weiter gezeigt, dass der ASD - Agile Systems Design Ansatz die notwendigen Methoden und Denkwerkzeuge für eine Prozessoptimierung bereitstellen kann. Jedoch ist ein hoher Individualisierungsgrad bei der Erarbeitung einer Gesamtmethode notwendig.

\section{Zusammenfassung und Ausblick}

Die Unternehmen sind mit einem zunehmenden Konkurrenzdruck und einer dynamischen Marktsituation konfrontiert. Um wettbewerbsfähig zu bleiben, steigt auch die Bereitschaft für die Veränderungen von bestehenden Prozessen. Für den Anwendungsfall sind jedoch weder rein plangetriebene Prozesse noch komplett agile Ansätze zielführend. Der ASD - Agile Systems Design Ansatz nach Albers stellt eine ganzheitliche Methodik zur Verfügung, welche die jeweilige Situation der Organisationseinheit in die Betrachtung der Prozessverbesserung mit einbezieht. Somit wird der Fokus auf 
den individuellen Handlungsbedarf gelegt. Im Sinne des ASD - Agile Systems Design werden durch methodische Unterstützung die Verbesserung der agilen Fähigkeiten angestrebt. Ein spezifisches Methodenprofil unterstützt bei der Realisierung von Prozessverbesserungen. Die Schwierigkeit hierbei ist je nach Bedarf geeignete strukturierende und flexible Elemente für den Anwendungsfall zu erarbeiten und diese zu einer Gesamtmethode zu kombinieren. Dabei muss der richtige Grad an Agilität gefunden werden, um die notwendige Flexibilität zu bieten und gleichzeitig die vorgegebenen Randbedingungen, die festen (Qualitäts-) Standards, die Richtlinien sowie den definierten In- und Output von und zu Nachbarprozessen einhalten zu können. Mehr noch muss die Akzeptanz für flexible Elemente und Handlungsweisen erreicht werden, sodass die Verbesserungspotenziale erkannt und nicht als bürokratischer Mehraufwand und Dokumentationspflicht verstanden werden.

Forschungsfrage 1: Wie gestaltet sich das Methodenprofil einer agilen Methodik für das technische Änderungsmanagement?

Durch die methodische Unterstützung des ASD - Agile Systems Design konnte die vorherrschende Situation in der Untersuchungsumgebung erfasst und das Problem eingegrenzt werden. Somit bildet das Methodenprofil und die damit verbundene Einschränkung des Lösungsraums die Basis für eine Prozessoptimierung. Mit Hilfe des Methodenprofils, des aufgebauten Prozessverständnisses und der situationsspezifischen Relevanz der ASD-Grundprinzipien können für die Funktionsbeschreibungen der Einflussfaktoren sinnvolle Methoden gefunden werden. Es konnte gezeigt werden, dass durch eine agil-strukturierende Prozesslösung das notwendige Maß an Flexibilität und Reaktionsfähigkeit zur Verfügung steht, aber dennoch die Randbedingungen berücksichtigt werden. Somit sind sowohl die Eingangsinformationen, die konkreten Aufgaben als auch die Umsetzung und Entscheidungen für die einzelnen Prozessschritte klar ersichtlich. Die gewählten Methoden und Elemente bieten für sich oder in Kombination die bedarfsgerechte Unterstützung der Prozessschritte.

Forschungsfrage 2: Welche Implikationen für das methodische Erarbeiten individueller Prozesslösungen lassen sich aus dem vorliegenden Anwendungsfall ableiten?

Der Anwendungsfall hat gezeigt, dass die methodische Herangehensweise für eine ganzheitliche Betrachtung einer Prozessoptimierung sinnvoll und hilfreich ist. Der Ansatz ASD - Agile Systems Design ist in der Lage sowohl einen Überblick über die vorherrschende Situation zu erarbeiten und den Lösungsraum einzuschränken als auch relevante Einflussfaktoren und Ziele zur Steigerung der agilen Fähigkeiten zu identifizieren. Jedoch ist eine anwendungsfallspezifische Betrachtung und eine individuelle methodische Erarbeitung einer Prozesslösung für den jeweiligen Kontext erforderlich. 
Zentraler Bestandteil der methodischen Erarbeitung ist ein möglichst umfangreiches Problemverständnis. Hierdurch kann ein höherer Grad an Vollständigkeit erreicht werden als ohne eine methodische Unterstützung. Zusätzliche Implikationen für das methodische Erarbeiten individueller Prozesslösungen wurden durch die Anforderungen an die Methode im Anwendungsfall identifiziert und aufgezeigt. Diese Erkenntnisse über bereits erfüllbare Anforderungen und Bereiche für Verbesserungspotenziale sollten in der weiteren Forschungsarbeit betrachtet und analysiert werden.

Für die Entwicklung einer individuellen Prozesslösung können mit Hilfe des ASD - Agile Systems Design Ansatzes sinnvolle Elemente empfohlen werden, die je nach Situation den Prozess bedarfsgerecht unterstützen können. Für das methodische Vorgehen wurde die Evaluierung der Methode beschrieben. Viele relevante Anforderungen an die Methode können bereits erfüllt werden. Weiteren Forschungsbedarf haben vor allem die in Abbildung 5 rot markierten Anforderungen. Hierbei soll zum einen ein Reporting über den Projektfortschritt ermöglicht werden und zum anderen sollen relevante Kriterien gemessen werden können. Diese Anforderungen bieten Ansatzpunkte für mögliche Verbesserungen des methodischen Vorgehens. Für den Anwendungsfall sind zusätzlich die nicht markierten (weiß) Anforderungen relevant und sollten in den weiteren Forschungsbedarf mit einfließen.

\section{Literaturverzeichnis}

Albers, A., Heimicke, J., Spadinger, M., Reiß, N., Breitschuh, J., Richter, T. et al. (2019). Eine Systematik zur situationsadäquaten Mechatroniksystementwicklung durch ASD - Agile Systems Design. KIT Scientific Working Papers, 113. Verfügbar unter http://nbn-resolving.de/urn:nbn:de:swb:90-918475

Albers, A., Heimicke, J. \& Trost, S. (2020). Effects and Interactions of agile Principles in the Process of Mechatronic System Development: Building a basic Understanding for adaptive Process Design. In Proceedings NordDesign 2020, n.p. https://doi.org/10.35199/NORDDESIGN2020.22

Albers, A., Heimicke, J., Trost, S. \& Spadinger, M. (2020). Alignment of the change to agile through method-supported evaluation of agile principles in physical product development. Proceedings of 30th CIRP Design Conference, 1-15.

Albers, A., Heimicke, J., Walter, B., Basedow, G. N., Reiß, N., Heitger, N. et al. (2018). Product Profiles: Modelling customer benefits as a foundation to bring inventions to innovations. Procedia CIRP, 70 (1), 253-258.

Albers, A., Reiß, N., Bursac, N. \& Breitschuh, J. (2016). 15 Years of SPALTEN Problem Solving Methodology in Product Development. In C. Boks (Ed.), Proceedings of NordDesign 2016. August 10-12, 2016, Trondheim, Norway (S. 411-420). Bristol, United Kingdom: The Design Society. Zugriff am 06.09.2017.

Baader, A., Montanus, S. \& Sfat, R. (2006). After Sales Service - mit produktbegleitenden Dienstleistungen profitabel wachsen. In K. Barkawi, A. Baader \& S. Montanus (Hrsg.), Erfolgreich mit After Sales Services. Geschäftsstrategien für Servicemanagement und Ersatzteillogistik (S. 3-14). Berlin, Heidelberg: Springer-Verlag Berlin Heidelberg. 
Blessing, L. T. \& Chakrabarti, A. (2009). DRM, a Design Research Methodology. London: Springer London.

Böhm, J. (2019). Erfolgsfaktor Agilität. Wiesbaden: Springer Fachmedien Wiesbaden.

Cooper, R. (1990). Stage-Gate Systems: A New Tool for Managing New Products. Business Horizons, 33, 44-54.

Dimitrova, D. (2009). Das Konzept der Metakompetenz. Wiesbaden: Gabler.

Frenz, W. (2020). Handbuch Industrie 4.0: Recht, Technik, Gesellschaft. Berlin, Heidelberg: Springer Berlin Heidelberg.

Gericke, K., Meißner, M. \& Paetzold, K. (2013). Understanding the context of product development. Proceedings of the 19th International Conference on Engineering Design (ICED13) Design For Harmonies, 75 (3).

Hales, C. \& Gooch, S. (2004). Managing Engineering Design (Second Edition). London: Springer.

Heimicke, J., Dühr, K., Krüger, M., Ng, G.-L. \& Albers, A. (2021). A Framework for Generating agile Methods for Product Development. In Proceedings of 31st CIRP Design Conference 2021 (in print).

Heimicke, J., Ng , G.-L., Krüger, M. \& Albers, A. (2021). A Systematic for Realizing Agile Principles in the Process of Mechatronic Systems Development through Individual Selection of Suitable Process Models, Development Methods and Practices. In Proceedings of 31st CIRP Design Conference 2021 (in print).

Jakoby, W. (2015). Projektmanagement für Ingenieure. Wiesbaden: Springer Fachmedien Wiesbaden.

Jania, T. (2004). Änderungsmanagement auf Basis eines integrierten Prozess- und Produktdatenmodells mit dem Ziel einer durchgängigen Komplexitätsbewertung. Dissertation, Universität Parderborn.

Kusay-Merkle, U. (2018). Agiles Projektmanagement im Berufsalltag. Berlin, Heidelberg: Springer Berlin Heidelberg.

Langer, S. (2016). Änderungsmanagement. In U. Lindemann (Hrsg.), Handbuch Produktentwicklung (S. 513-539). München: Carl Hanser Verlag GmbH \& Co. KG.

Reiß, N. (2018). Ansätze zur Steigerung der Methodenakzeptanz in agilen Prozessen der PGE -Produktgenerationsentwicklung, Karlsruher Institut für Technologie (KIT).

Schütze, R. (1994). Kundenzufriedenheit. After-Sales-Marketing auf industriellen Märkten (Neue betriebswirtschaftliche Forschung, Bd. 91, Nachdr). Zugl.: Berlin, Freie Univ., Diss., 1991. Wiesbaden: Gabler.

Siebler-Guth, F. (2019). Der Prozess mobiler Entwicklungsprojekte: Muster agiler Methoden. Wiesbaden: Springer Fachmedien Wiesbaden. 


\section{Kontakt}

Jonas Heimicke, M. Sc.

Univ.-Prof. Dr.-Ing. Dr. h. c. Albert Albers

IPEK - Institut für Produktentwicklung am Karlsruher Institut für Technologie (KIT)

Kaiserstraße 10

76131 Karlsruhe

www.kit.edu 